\title{
ANTIBACTERIAL ACTIVITY OF APIS FLOREA HONEY.
}

\author{
Sulbha V. Kulkarni ${ }^{1}$, Shilpa Pawankar', and Mayuri Kulkarni ${ }^{2}$ \\ 1 Sevadal Mahila Mahavidyalaya, Nagpur. \\ 2 Sharad Pawar Dental College, Savangi Meghe, Wardha. \\ Corresponding author Email : drsulbhakulkarni@gmail.com
}

\begin{abstract}
:
Three honey samples were used to study the antibacterial activity. Few gram positive and gram negative bacteria viz. Lactobacillus lactis, Staphylococcus aureus, Escherichia coli (2685 and 5051) and Pseudomonas aureginosa (5029 and 2200) were used. In the present work antibacterial activity was studied by using disc diffusion method and determined as an equivalent of the inhibition zone diameters (in millimeters) after incubation of the culture at $37^{\circ} \mathrm{C}$ for twenty four hours for bacterial species. Pure honey and their dilutions had good results in Staphylococcus aureus, Escherichia coli (5051) and Pseudomonas aureginosa (5029 and 2200) while pure honey and their dilutions had no effect on Lactobacillus lactis and Escherichia coli (2685).
\end{abstract}

\section{Keywords:}

Key words: Apis florea, Honey, Antibacterial activity, Disc diffusion method.

\section{Introduction:}

Introduction: According to World Health Organization estimates, some 80 percent of people living in developing countries rely on harvested wild plants for their primary health care. In the developing world the use of antibiotics constitute a sizable fraction of medicines consumed. However, because of the microbes turning resistant especially to the synthetic antibiotics in use, the effectiveness of the antibiotics has been diminishing World Health Organization (1999). This type of resistance to antimicrobial agent has already become a serious issue in many areas of the world especially in developing countries (Assefa and Yohannes, 1997). One of the popular antimicrobial natural substances described in Ayurveda as a potent medicine for several uses is honey. The antimicrobial properties of honey have been known to humans for centuries. Honey has been reported to have an inhibitory effect on number of Aerobes, Anaerobes, Gram positive and Gram negatives bacteria. Honey 
consists of various constituents such as water, carbohydrates, proteins, vitamins, amino acids, energy and minerals. Besides the major ones, there must also be several minor constituent in honey, which may be playing a key role in determining the antibacterial behavior of honey. In the present study, such natural antibiotic-honey is used to study their antibiotic activity against six Lactobacillus lactis, Staphylococcus aureus, Escherichia coli (2685 and 5051) and Pseudomonas aureginosa (5029 and 2200).

\section{Material and Method:}

Materials and Methods: Eighteen honey samples of Apis florea were collected during winter from Nagpur region and kept in dark air tight containers. Three honey samples of different region were taken for preparing honey solutions of different concentrations viz. 75\%, 50\% and 25\% were constituted by adding distilled water to honey. To assay the antibacterial activity of honey six strains of bacteria- Escherichia coli (NCIM 2685 and 5051), Pseudomonas aeruginosa (NCIM 5029 and 2200), Lactobacillus lactis (NCIM 2370) and Staphylococcus aureus (NCIM 2127) were utilized. Well diffusion method was employed to test the antibacterial activity. Bacteria were cultured on the nutrient agar prior to transfer into nutrient broth. For bacterial culture, a loopful of culture was picked from the nutrient agar culture and inoculated into nutrient broth and incubated for 24 hours at $37^{\circ}$. Density of bacterial strains was measured with Mc Farland"s standard solution. The size was adjusted to 0.5 Mc Farland"s standard turbidity, approximately 108 colony forming units (CFU/ml). The cell suspension $(100 \mu 1$ target) strains were introduced into the nutrient agar and spread thinly on the plate using a glass spreader. Wells of $8 \mathrm{~mm}$ diameter were made by borer. Different concentrations of honey were poured in the well respectively. The plates were incubated at $37^{\circ} \mathrm{c}$ for 24 hours. Octadisc of +ve, ve and combo were used as a control. The diameter of the inhibition zones around the disc were measured in millimeter after 24 hours. Tests were performed in duplicate. 


\section{Result and Discussion:}

The therapeutic properties of honey have been described for centuries and honey is traditionally used by many different cultures around the world. The result of present work was presented in Table-I. A number of key properties of honey have been described and supported in the scientific literature including the antimicrobial and the immune stimulatory activity of honey. The important conclusion which can be drawn from the present study was that honey has broad spectrum antibacterial activity. Three honey samples of Apis florea i.e. LH-1, LH-2 and LH-3 were used to inhibit the activity of six bacteria (two species of Gram-positive: Lactobacillus lactis, Staphylococcus aureus and four species of Gram-negative :E.coli 2685,5051; Pseudomonas aeruginosa 5029, 2200) Zones of inhibition was observed in some gram-positive and gram negative bacteria Staphylococcus aureus, E.coli (5051) in all concentration of honey except $100 \%$ and $25 \%$ solution of honey sample in LH-2 respectively (Ahmed Shawki Ibrahim, Assadi-Pooya et al. 2003,Nuriza et al., 2005). Whereas zones of inhibition in $100 \%$ of honey was not observed in E.coli (2685), Lactobacillus lactis and S. aureus (Muli, et al. 2008; Abhishek et al., 2010). Honey concentrations of all samples also showed good result against Pseudomonas aureginosa (5029) and in P. aureginosa (2200) in honey sample 02 but zones of inhibition was least Pseudomonas aureginosa (5029) in 100\% and $75 \%$ of honey sample (LH-1,2)solution (Ahmed Shawki Ibrahim, AssadiPooya et al. 2003,Nuriza et al., 2005). Zones of inhibition were not observed in Pseudomonas aureginosa (2200) in all concentrations of honey samples 01 and 03 (Muli, et al. 2008)).

\section{Conclusion:}

Pure honey A. florea had more effect in inhibiting the bacterial growth than different dilutions of honey. Among the different samples of honey used in the study, most of the honey samples of Apis florea showed maximum antibacterial activity as compare to other honey samples of Apis florea. Further, this study 
also showed that like antibiotics, some organisms were sensitive to some honey samples while other was insensitive.

\section{Acknowledgement:}

Authors are thankful to UGC Delhi for funding project on honey and also thankful to Hon"ble Principal Dr. P.N. Charde for support during work.

\section{Reference:}

Abhishek Chauhan, Vimlendu Pandey, K. M.Chacko and R. K.Khandal (2010): Antibacterial activity of Raw and Processed honey . Electronic Journal of Biology, 2010, Vol. 5(3):58-56.

Ahmed Shawki Ibrahim: Antibacterial action of honey. Science ASADI-POOYA, A. A., PNJEHSHAHIN, M. R. \& BESESHTI, S. (2003): The antimycobacterial effect of honey: An in vitro study, Rivista di Biology, 96:491-495.

Assefa A., Yohannes G. (1997): Antibiotic sensitivity of S. aureus and E. coli strains isolated in Gondar, Ethiopia, Tropical Doctor, 27(2): 121-126.

E. M. Muli, J. M. Maingi, J. Macharia (2008): Antimicrobial properties of Propolis and Honey from Kenyan Stingless bee, Dactylurina Schimidti. APIACTA 43 (2008).

Nuriza Tumin, N. Arsyiah A. Halim, M. Shahjahan, Noor Izani N. J., Munavvar A, Satter, Abdul Hye Khan and S. S. J. Mohsin 2005: Antibacterial activity of Local Malaysian Honey. Malaysian Journal of Pharmaceutical Sciences, Vol. 3, No. 2, 1-10 (2005) World Health Organization. (1999) Drug information WHO, Geneva, 13(4): 230-233. 
Table: I

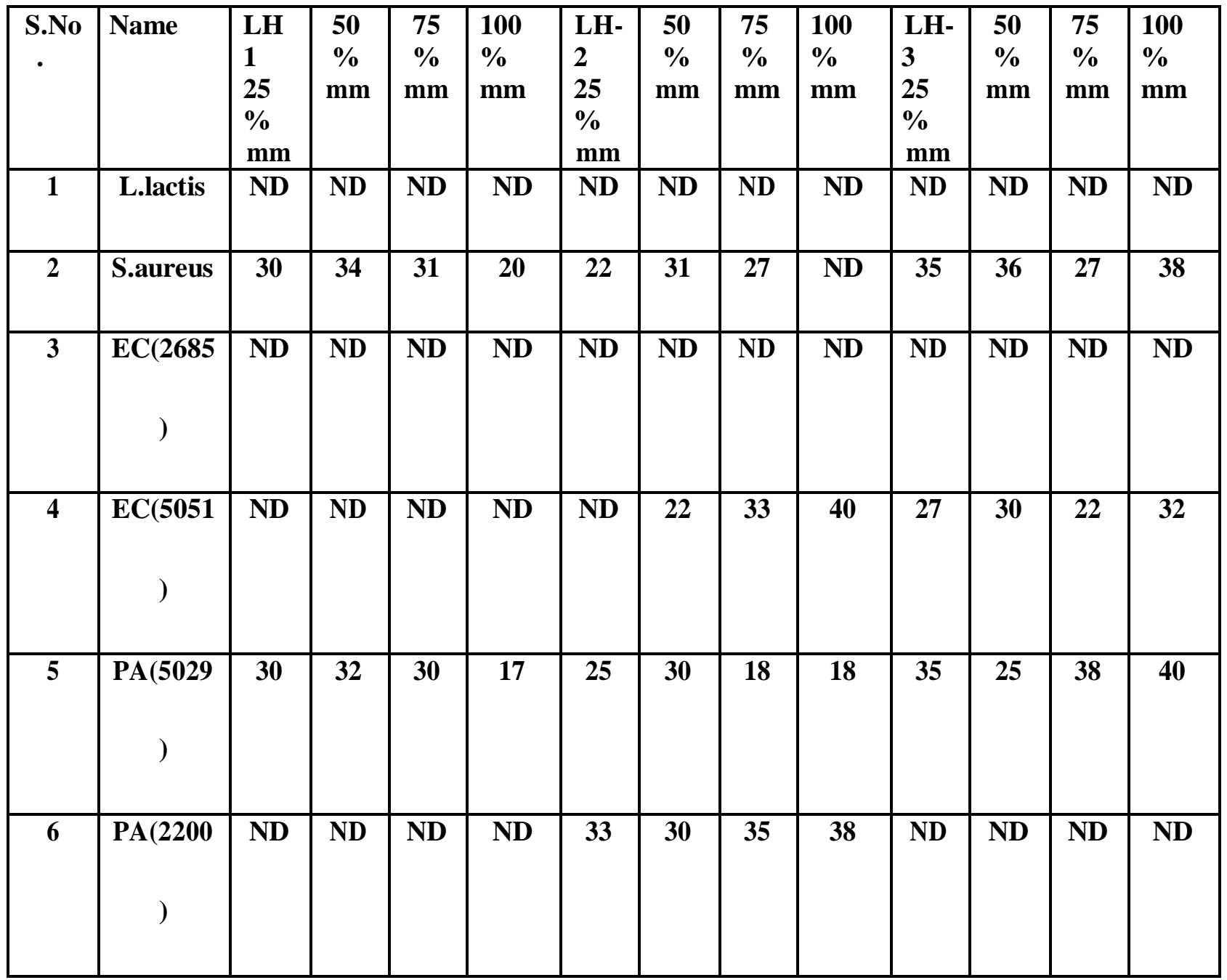




\section{Photoplate: I}

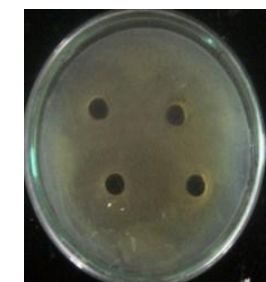

WH-39 Staphylococcus

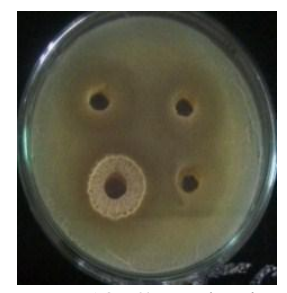

WH-58 Staphylococcus

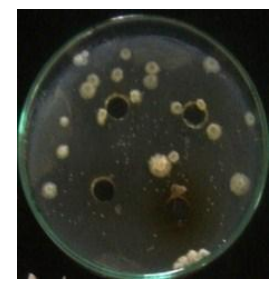

WH-58 P.aeruginosa (2200)

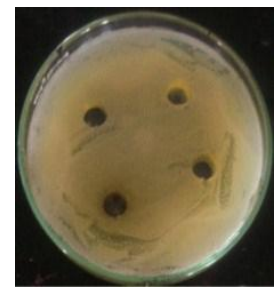

WH-41 Staphylococcus

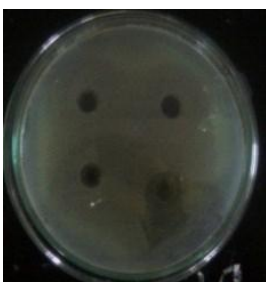

WH-39 P.aeruginosa (5029)

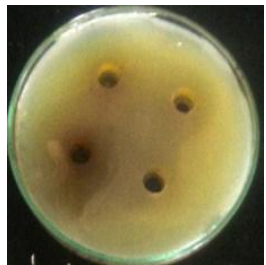

WH-58 P.aeruginosa (5029)

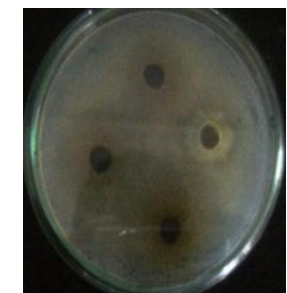

WH-58 E.coli (5051)

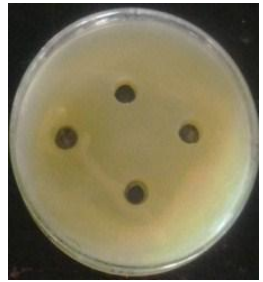

WH-41-P.aeruginosa (5029)

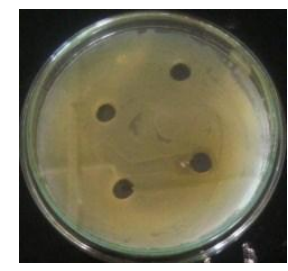

WH-41 E.coli (5051) 